\title{
OPTIMISATION DU RENDEMENT ET DE LA TEMPÉRATURE D'UN CAPTEUR SOLAIRE PLAN À EAU PAR SIMULATION
}

\author{
Kanouté, Y. ${ }^{1}$, Traoré, I. ${ }^{1}{ }^{*}$, Sanogo, S. ${ }^{1}$, Aroudam, E. ${ }^{2}$, and Ba, A. ${ }^{1}$ \\ ${ }^{1}$ Laboratoire d'Optique de Spectroscopie et des Sciences Atmosphériques, Faculté des Sciences et Techniques BP : E 3206. \\ Département de Physique - Bamako \\ ${ }^{2}$ Laboratoire de mécanique et simulation des systèmes mécaniques, Université Abdelmalek Essaâdi, Tétouan, Maroc \\ *traoreamenophis@gmail.com
}

\section{INFOS SUR L'A R T I C L E \\ Historique de l'article: \\ Reçu le : 26 novembre 2020 \\ Reçu en format revisé le : 09 février 2021 \\ Accepté le : 12 février 2021}

Mots-Clés : capteur solaire plan à eau, rendement thermique, simulation, optimisatin, transfert thermique.

\begin{abstract}
R E S U M E / A B S T R A C T
Cette étude consiste à la modélisation et à l'application de méthodes numériques afin d'optimiser les performances d'un capteur solaire plan à eau sous les conditions météorologiques du Mali, particulièrement au mois de janvier ou l'ensoleillement est faible et la consommation en eau chaude importante. Les résultats obtenus montrent que lorsque la vitesse du vent passe de $1 \mathrm{~m} / \mathrm{s}$ à $4 \mathrm{~m} / \mathrm{s}$ le rendement décroit de $69 \%$ à $65 \%$ pour une intensité du rayonnement solaire de $460 \mathrm{~W} / \mathrm{m}^{2}$. L'augmentation de l'épaisseur de l'isolant, des diamètres intérieur et extérieur des tubes donne un rendement pouvant atteindre respectivement $67 \%$ et $70 \%$. Lorsque le matériau constituant l'absorbeur, la vitre et l'isolant sont respectivement en acier chrome, en poly-méthacrylate, en mousse de polyuréthane, on obtient des rendements de $71 \%, 61,5 \%$ et 52,5\% respectivement pour une intensité du rayonnement solaire de $460 \mathrm{~W} / \mathrm{m}^{2}$. Aussi, cette étude a montré que les paramètres externes tels que le rayonnement solaire, la vitesse du vent et internes (propriétés optiques et thermo-physiques des différents composants du capteur) influent très peu sur la température du fluide caloporteur.
\end{abstract}

\section{INTRODUCTION}

Les combustibles fossiles utilisés pour la production de l'énergie tendent à s'épuiser au vu de la demande croissante d'énergie au niveau mondiale. Cette énorme consommation d'énergie résultant du développement économique, social et de l'augmentation de la population est en train de produire des effets négatifs sur notre environnement. Ces effets se manifestent principalement à travers les émissions de gaz à effet de serre, la pollution, la déforestation, la dégradation des terres et des ressources naturelles. Dans ce contexte, les pays africains, n'ont plus le choix que de diversifier leurs sources d'énergie. Les énergies renouvelables apparaissent alors comme une alternative. Ces énergies qui utilisent des ressources naturelles tels que le soleil, le vent, l'eau, la biomasse sont propres, inépuisables et disponibles. Parmi ces énergies renouvelables, le plus abondant qu'est le soleil peut être exploité dans plusieurs domaines comme la production d'électricité, la cuisson solaire, le chauffage, la production d'eau chaude.

En effet, l'utilisation de l'énergie solaire la plus simple et la plus directe, est la production d'eau chaude à destination des ménages et des bâtiments publics. Au Mali, le chauffage de l'eau via le solaire est très peu utilisé à causes de plusieurs contraintes dont le coût élevé des installations, la méconnaissance de ce type de dispositif par la population et surtout l'aridité du climat caractérisé par une période de froid (2 mois/an au maximum) qui nécessite un faible besoin en eau chaude dans les habitations. Le gisement solaire du Mali est considérable, largement inexploité avec une irradiation solaire très importante (en moyenne $6 \mathrm{kWh} / \mathrm{m}^{2} / \mathrm{j}$ ), répartie sur l'ensemble du territoire pour une durée d'ensoleillement journalier de 7 à 10 heures (Ministère de l'énergie et de l'eau., 2020). L'exploitation de l'énergie solaire pour chauffer l'eau nécessite des dispositifs qui permettent de convertir le rayonnement solaire incident sur la surface de la terre, tels que les panneaux photovoltaïques, les capteurs plans, les capteurs à tube sous vide, les capteurs à concentrateurs. Plusieurs travaux ont été effectués sur l'optimisation des capteurs solaires plans, ayant surtout pour but l'amélioration de son rendement instantané qui est la performance la plus significative (Hhedim., 2003 ; Benkhelifa., 2098 ;

Sandali et Korti., 2016). Selmi et al., 2008 ont montré que les méthodes de simulation numérique pouvaient être appliquées à l'étude des capteurs solaires et obtenir des résultats très proches de l'expérience.

L'objectif de ce travail consiste à la modélisation et à l'application de méthodes numériques afin d'optimiser les performances d'un capteur solaire plan à eau sous les conditions météorologiques du Mali, particulièrement au mois de janvier ou l'ensoleillement est très faible et la consommation en eau chaude importante. 
L'effet des paramètres tels que la vitesse du vent, l'épaisseur et la nature de l'isolant, les dimensions des tubes, la nature de l'absorbeur, la nature de la vitre influant sur le rendement et la température du capteur solaire plan seront étudiés afin de servir de référence pour une production locale et aussi permettre aux particuliers de faire un choix approprié.

\section{DISPOSITIF À ÉTUDIER}

Le capteur solaire plan qui sera modélisé dans notre travail est schématisé par la figure 1. Il comprend :

- Une couverture transparente (vitrage) faite en matériaux transparents au rayonnement visible mais opaque au rayonnement infrarouge permettant de réaliser un effet de serre; elle protège aussi l'intérieur du capteur contre les effets de l'environnement.

- Un absorbeur qui absorbe le rayonnement solaire de courtes longueurs d'onde et le convertit en chaleur. Il est généralement peint en noir pour absorber pratiquement tous les rayonnements dans le spectre du visible. Il est constitué d'une plaque à laquelle sont intégrés des tubes à travers lesquels circule le fluide caloporteur.

- Un fluide caloporteur chargé de transporter la chaleur emmagasinée par l'absorbeur vers la source de température.

- Une isolation thermique servant à limiter les déperditions thermiques du capteur sur les côtés arrière et latérale.

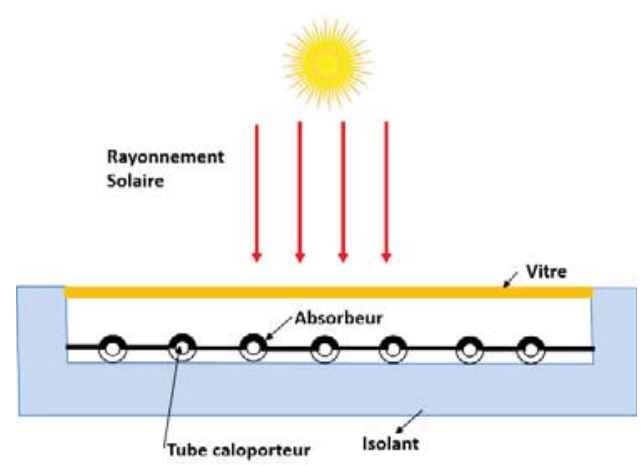

Fig.1. Schéma du capteur solaire plan

\section{MODÉLISATION ET SIMULATION NUMÉRIQUE DU CAPTEUR SOLAIRE PLAN}

\subsection{Hypothèses}

Pour simplifier notre étude, les hypothèses ci-dessous ont étés considérées :

- le soleil est assimilé à un corps noir.

- La surface du capteur est uniformément éclairée.

- Le transfert de chaleur à une seule dimension à travers les couches du système.

- Le débit massique est uniforme dans les tubes du capteur.

- Le transfert de chaleur à partir des bords du capteur est négligeable.

- La vitesse du vent extérieur est supposée de direction toujours parallèle aux faces du capteur.
- Le flux de chaleur reçu par le capteur est fonction du temps.

- Les propriétés physiques des matériaux ne sont pas fonction de la température.

- Les propriétés physiques du fluide ne sont pas fonction de la température.

- La poussière et la saleté sur le collecteur sont négligeables.

- Le régime d'écoulement est transitoire.

- La température de la plaque absorbante est supposée égale à celle des tubes.

- La température du sol est supposée égale à la température ambiante.

\subsection{Bilan thermique des composants du capteur} solaire plan

\section{a) Bilan énergétique de la couverture transparente (vitre)}

- Flux de chaleur incident et absorbé par la vitre.

$$
\mathrm{Q}_{\mathrm{V}}=\alpha_{1} \mathrm{~S}_{1} \mathrm{I}_{\mathrm{C}}
$$

Où $\alpha_{1}, S_{1}, I_{C}$ représentent respectivement le coefficient d'absorption de la vitre, la surface de la vitre $\left(\mathrm{m}^{2}\right)$ et l'éclairement global incident sur le capteur plan $\left(\mathrm{W} / \mathrm{m}^{2}\right)$.

- Flux de chaleur échangé par convection entre le vitrage et l'air ambiant.

$$
\mathrm{Q}_{\mathrm{clam}}=\mathrm{h}_{\mathrm{clam}} \mathrm{S}_{1}\left(\mathrm{~T}_{1}-\mathrm{T}_{\mathrm{am}}\right)
$$

$\mathrm{T}_{1}, \mathrm{~T}_{\mathrm{am}}, \mathrm{h}_{\mathrm{clam}}$ désignant respectivement la température de la vitre $(\mathrm{K})$, la température ambiante $(\mathrm{K})$, le coefficient d'échange par convection entre la vitre et l'air ambiant $\left(\mathrm{W} / \mathrm{m}^{2} \mathrm{~K}\right)$.

$\mathrm{h}_{\mathrm{clam}}$ est donné par la correlation de MacAdams (McADAMS., 2054).

- Flux de chaleur échangé par rayonnement entre le vitrage et le ciel.

$$
\mathrm{Q}_{\mathrm{r} 1 \text { ciel }}=\mathrm{h}_{\mathrm{r} 1 \text { ciel }} \mathrm{S}_{1}\left(\mathrm{~T}_{1}-\mathrm{T}_{\text {ciel }}\right)
$$

avec $h_{r 1 \text { ciel }}=\varepsilon_{1} \sigma\left(T_{1}^{2}+T_{\text {ciel }}^{2}\right)\left(T_{1}+T_{\text {ciel }}\right)$ le coefficient d'échange par rayonnement entre la vitre et le ciel $\left(\mathrm{W} / \mathrm{m}^{2} \mathrm{~K}\right) ; \varepsilon_{1}$ l'émissivité du vitrage et $\sigma$ la constante de Stéphane Boltzman.

La température du ciel $\left(\mathrm{T}_{\text {ciel }}\right)$ est estimée par la correlation de Swinbank (Duffie et William., 2080).

- Flux de chaleur échangé par convection entre le vitrage et l'absorbeur

$$
\mathrm{Q}_{\mathrm{c} 12}=\mathrm{h}_{\mathrm{c} 12} \mathrm{~S}_{2}\left(\mathrm{~T}_{2}-\mathrm{T}_{1}\right)
$$

avec $h_{C 12}=N_{U} \frac{\lambda_{\text {air }}}{L_{C}} ;$ où $\mathrm{S}_{2}, \mathrm{~T}_{2}, \mathrm{~L}_{\mathrm{C}}, \lambda_{\text {air }}, \mathrm{h}_{\mathrm{c} 12}$ désignant respectivement la surface de l'absorbeur $\left(\mathrm{m}^{2}\right)$, la température de l'absorbeur $(\mathrm{K})$, la distance entre la vitre et l'absorbeur (m), la conductivité thermique de l'air (W/ $\mathrm{m} \mathrm{K}$ ), le coefficient d'échange thermique par convection entre la vitre et l'absorbeur $\left(\mathrm{W} / \mathrm{m}^{2} \mathrm{~K}\right)$. 
Le nombre de Nusselt $\mathrm{Nu}$ pour deux surfaces parallèles inclinées d'un angle entre $0^{\circ}$ et $75^{\circ}$, est donné par la corrélation de Hollands et al., 2076.

- Flux de chaleur échangé par rayonnement entre le vitrage et l'absorbeur.

$$
\mathrm{Q}_{\mathrm{r} 12}=\mathrm{h}_{\mathrm{r} 12} \mathrm{~S}_{2}\left(\mathrm{~T}_{2}-\mathrm{T}_{1}\right)
$$

$$
\text { où } h_{r 12}=\sigma \frac{\left(T_{1}+T_{2}\right)\left(T_{1}^{2}+T_{2}^{2}\right)}{\frac{1}{\varepsilon_{2}}+\frac{1}{\varepsilon_{1}}-1}
$$

$\varepsilon_{2}$ : émissivité de l'absorbeur ;

$h_{r 12}$ : coefficient d'échange thermique par rayonnement entre la vitre et l'absorbeur $\left(\mathrm{W} / \mathrm{m}^{2} \mathrm{~K}\right)$.

Le bilan énergétique de la couverture transparente est :

$$
\begin{aligned}
m_{1} c_{1} \frac{d T_{1}}{d t}= & \alpha_{1} S_{1} I_{c}+S_{2}\left(h_{c 12}+h_{r 12}\right)\left(T_{2}-T_{1}\right) \\
& -h_{\text {c1am }} S_{1}\left(T_{1}-T_{a m}\right)-h_{r 1 \text { ciel }} S_{1}\left(T_{1}-T_{\text {ciel }}\right)
\end{aligned}
$$

\section{b) Bilan énergétique de la plaque absorbante}

- Flux de chaleur incident reçu par l'absorbeur

$$
\mathrm{Q}_{\mathrm{ab}}=\alpha_{2} \mathrm{~S}_{2} \tau_{1} \mathrm{I}_{\mathrm{C}}
$$

$\alpha_{2}$ : coefficient d'absorption de l'absorbeur et $\tau_{1}$ : coefficient de transmission de la vitre

- Flux de chaleur échangé par convection entre l'absorbeur et le fluide caloporteur (eau)

$$
\mathrm{Q}_{\mathrm{C} 23}=\mathrm{h}_{\mathrm{C} 23} \mathrm{~S}_{23}\left(\mathrm{~T}_{2}-\mathrm{T}_{3}\right)
$$

avec

$$
h_{C 23}=N_{U} \frac{\lambda_{23}}{d_{23}}
$$

$h_{c 23}$ : coefficient d'échange par convection entre l'absorbeur et le fluide caloporteur $\left(\mathrm{W} / \mathrm{m}^{2} \mathrm{~K}\right)$.

$\mathrm{T}_{3}$ : température du fluide caloporteur $(\mathrm{K})$.

$\mathrm{S}_{23}$ : surface de contact absorbeur fluide caloporteur $\left(\mathrm{m}^{2}\right)$ $\mathrm{d}_{23}$ : diamètre intérieur du tube $(\mathrm{m})$.

$\lambda_{23}$ : conductivité thermique du fluide caloporteur $\left(\mathrm{W} / \mathrm{m}^{-1}\right.$ $\left.\mathrm{K}^{-1}\right)$.

$\mathrm{h}_{\mathrm{C} 23}$ : coefficient d'échange par convection entre l'absorbeur et le fluide caloporteur $\left(\mathrm{W} / \mathrm{m}^{2} \mathrm{~K}\right)$.

- Flux de chaleur échangé par conduction entre l'absorbeur et l'isolant

$$
\mathrm{Q}_{\mathrm{d} 24}=\left(\psi_{1}+\psi_{2}\right)\left(\mathrm{T}_{2}-\mathrm{T}_{\text {isl }}\right)
$$

Avec

$$
\psi_{1}=\frac{1}{\frac{e_{2}}{s_{a b i} \lambda_{i s l}}+\frac{e_{i s l}}{s_{a b i} \lambda_{a b s}}}
$$

et

$$
\psi_{2}=\frac{1}{\frac{e_{2}}{s_{l a} \lambda_{l a}}+\frac{e_{l a}}{s_{l a} \lambda_{a b s}}}
$$

$\psi_{2}, \psi_{1}$ sont de constantes et $\mathrm{T}_{\text {isl }}, \lambda_{\text {isl }}, \lambda_{\mathrm{abs}}, \lambda_{\mathrm{la}}, \mathrm{S}_{\mathrm{la}}, \mathrm{S}_{\mathrm{abi}}, \mathrm{e}_{2}$, $\mathrm{e}_{\mathrm{la}}, \mathrm{e}_{\mathrm{isl}}$ représentent respectivement la température de l'isolant $(\mathrm{K})$, la conductivité thermique de l'isolant horizontal $\left(\mathrm{W} \cdot \mathrm{m}^{-1} \cdot \mathrm{K}^{-1}\right)$, la conductivité thermique de l'absorbeur $\left(\mathrm{W} \cdot \mathrm{m}^{-1} \cdot \mathrm{K}^{-1}\right)$, la conductivité thermique de l'isolant latéral $\left(\mathrm{W} \mathrm{m}^{-1} \mathrm{~K}^{-1}\right)$, la surface de contact entre l'absorbeur et l'isolant latéral $\left(\mathrm{m}^{2}\right)$, la surface de contact entre l'absorbeur et l'isolant horizontal $\left(\mathrm{m}^{2}\right)$, l'épaisseur de l'absorbeur (m), l'épaisseur de l'isolant latéral (m), l'épaisseur de l'isolant horizontal (m).

Le bilan final de la plaque énergétique est :

$$
\begin{gathered}
m_{2} c_{2} \frac{d T_{2}}{d t}=\alpha_{2} S_{2} \tau_{1} I_{c}-\left(\Psi_{1}+\Psi_{2}\right)\left(T_{2}-T_{i s l}\right) \\
-S_{2}\left(h_{c 12}+h_{r 12}\right)\left(T_{2}-T_{1}\right)-h_{c 23} S_{23}\left(T_{2}-T_{3}\right)
\end{gathered}
$$

\section{c) Bilan énergétique du fluide caloporteur}

- Flux de chaleur échangé par convection entre l'absorbeur et le fluide caloporteur.

$c_{3}\left(m_{3} \frac{d T_{3}}{d t}+\dot{m} \frac{d T_{3}}{d x}\right)=h_{c 23} S_{23}\left(T_{2}-T_{3}\right)$

$\dot{m}$ : débit massique du fluide caloporteur $(\mathrm{Kg} / \mathrm{s})$.

\section{d) Bilan énergétique de l'isolation}

- Flux de chaleur échangé par rayonnement entre l'isolant et le sol.

$$
Q_{i s l s}=h_{r i s} S_{i s}\left(T_{i s l}-T_{s o l}\right)
$$

Avec $_{h_{r i s}}=\sigma \varepsilon_{i s l}\left(T_{i s l}+T_{s o l}\right)\left(T_{i s l}^{2}+T_{s o l}^{2}\right)$

$\mathrm{T}_{\text {sol }}, \mathrm{h}_{\mathrm{ris}}, \varepsilon_{\mathrm{isl}}, \mathrm{S}_{\mathrm{is}}$ sont respectivement la température du sol $(\mathrm{K})$, le coefficient d'échange par rayonnement entre l'isolant et le sol $\left(\mathrm{W} / \mathrm{m}^{2} \mathrm{~K}\right)$, l'émissivité de l'isolant, la surface de l'isolant $\left(\mathrm{m}^{2}\right)$.

- Flux de chaleur échangé par convection entre l'isolant et l'air ambiant

$$
Q_{\text {ci } a}=h_{c i s} S_{i s}\left(T_{i s l}-T_{a m}\right)
$$

avec $h_{\text {cis }}=h_{\text {clam }}=5,67+3,86 \times V_{\text {vent }}$

$$
\mathrm{V}_{\text {vent }}=\text { vitesse du vent }
$$

$\mathrm{h}_{\text {cis }}$ : coefficient d'échange par convection entre l'isolant et l'air ambiant $\left(\mathrm{W} / \mathrm{m}^{2} \mathrm{~K}\right)$. Le bilan total de l'isolation donne :

$$
\begin{aligned}
m_{i s} c_{i s} \frac{d T_{i s l}}{d t}= & \left(\Psi_{1}+\Psi_{2}\right)\left(T_{2}-T_{i s l}\right)-h_{r i s} S_{i s}\left(T_{i s l}-T_{s o l}\right) \\
& -h_{c i s} S_{i s}\left(T_{i s l}-T_{a m}\right)
\end{aligned}
$$

Le bilan d'énergie total qui décrit le comportement thermique du capteur étudié est formé des équations (6) (10) (11) (15).

\subsection{Détermination des coefficients globaux d'échange de chaleur avec l'environnement}

Les pertes de chaleur se produisent vers l'avant, l'arrière et les parties latérales du capteur. Ces pertes se manifestent sous les trois modes de transfert de chaleur.

L'analogie électrique liée aux différentes résistances thermiques lors des échanges de chaleur effectués sur les différents éléments du capteur est illustrée sur la Figure 2. 

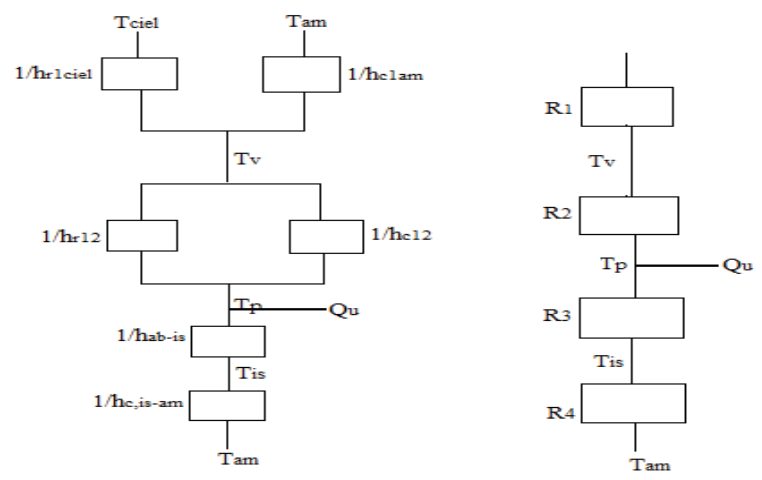

Fig.2 : Circuit électrique équivalent relative à un capteur solaire plan

a- Coefficient de pertes thermiques vers l'avant Le coefficient de pertes thermiques global vers l'avant du capteur est donné par la relation suivante :

$$
U_{a v}=\frac{1}{R_{1}+R_{1}}=\frac{1}{\frac{1}{h_{c 1 a m}+h_{r 1 c i e l}}+\frac{1}{h_{c 12}+h_{r 12}}}
$$

\section{b- Coefficient de pertes thermiques vers l'arrière} La valeur de ce coefficient est très petite comparée à celui des pertes vers l'avant, car le capteur est très bien isolé à l'arrière. Son expression est :

$$
U_{a r}=\frac{1}{R_{3}}=\frac{\lambda_{i s l}}{e_{i s l}}
$$

\section{c- Coefficient de pertes thermiques latérale}

Ce coefficient est inférieur à celui des pertes arrière, dans la mesure où la surface latérale du capteur est plus petite.

$$
U_{l a t}=\frac{\lambda_{i s l} \mathrm{~S}_{l a}}{e_{l a} \mathrm{~A}_{c}}
$$

$A_{c}$ : surface du capteur, exposée au rayonnement $\left(\mathrm{m}^{2}\right)$.

Le coefficient d'échange de pertes thermiques global vers l'environnement est la somme des trois coefficients (16), (17), (18).

\section{d- Rendement du capteur}

Le rendement instantané (ou thermique) du capteur solaire plan est égal au rapport entre le flux utile récupéré et l'éclairement global incident reçu par la surface du capteur.

$$
\eta_{c}=\frac{Q_{u}}{A_{c} I_{c}}
$$

$Q_{u}=\dot{m} c_{p f}\left(T_{f s}-T_{f e}\right)$

$\mathrm{Q}_{\mathrm{u}}$ : flux utile récupéré par le fluide caloporteur $(\mathrm{W})$.

$\mathrm{C}_{\mathrm{pf}}$ : Chaleur spécifique du fluide caloporteur (J/kg K).

$\mathrm{T}_{\mathrm{fs}}$ : Température de sortie du fluide $(\mathrm{K})$.

$\mathrm{T}_{\mathrm{fe}}$ : Température d'entrée du fluide $(\mathrm{K})$.

\subsection{Validation du code numérique}

Les températures des composantes ont été prises égales à la température ambiante (initialisation) à l'exception de la température de l'absorbeur qui est légèrement supérieure. Les données de température ambiante et de rayonnement solaire ont été recueillies le 01/01/2020 à la station météorologique de la faculté des sciences et techniques de Bamako.

Les équations décrivant les différents échanges de chaleur mis en jeu à l'intérieur d'un capteur en régime transitoire (6) (10) (11) (15) ont été discrétisées par la méthode de différence finie. Ces équations ont ensuite été résolues numériquement avec le logiciel Matlab (Matlab., 2020) par la méthode itérative de Gauss-Seidel.

La figure 3, montre l'évolution des différentes températures du capteur en fonction du temps. La température de l'absorbeur est la plus élevée. En effet, l'absorbeur absorbe la grande partie du rayonnement solaire grâce à son fort coefficient d'absorption pour chauffer le fluide. La température du fluide caloporteur est assez élevée par rapport aux autres composantes du capteur. L'écart entre les températures est principalement dû au coefficient de convection entre les composantes. La température de l'isolant est assez faible par rapport à celle $\mathrm{du}$ fluide parce qu'il résiste aux pertes thermiques vers les côtés de l'absorbeur ainsi qu'à celles dues à l'action du vent. La température de la vitre est faible par rapport aux autres composantes du capteur à cause des pertes thermiques vers l'extérieur et à son faible coefficient d'absorption. Des fluctuations dues aux rayonnements solaires apparaissent pendant les premières heures en accord avec le résultat de Brahimi., 2016.

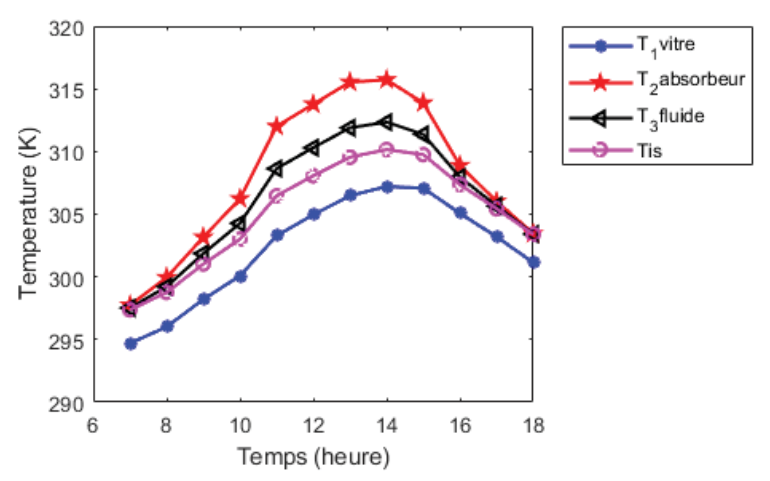

Fig.3. Evolution des Températures de différentes composantes du capteur en fonction du temps.

\section{RÉSULTATS ET DISCUSSION}

Les données météorologiques utilisées dans ce travail sont résumées dans le tableau ci-dessous

Table 1 : Paramètres d'entrée pour la simulation du capteur plan étudié

\begin{tabular}{|l|l|l|}
\hline Paramètre & Valeur & Référence \\
\hline Latitude Bamako & $12,65^{\circ}$ & \\
\hline Longitude Bamako & $-8^{\circ}$ & \\
\hline Altitude Bamako & $338 \mathrm{~m}$ & \\
\hline$V_{\text {vent }}:$ vitesse du vent & $3,5 \mathrm{~m} / \mathrm{s}$ & \\
\hline$\beta$ :L'angle d'inclinaison & $12,65^{\circ}$ & \\
\hline
\end{tabular}




\begin{tabular}{|c|c|c|}
\hline Paramètre & Valeur & Référence \\
\hline Dimension capteur (m) & $\begin{array}{l}1,950 \times 1,205 \\
\times 0,105\end{array}$ & \multirow{11}{*}{$\begin{array}{l}\text { (Nedjemedd } \\
\text { ine., } \\
2017)\end{array}$} \\
\hline $\mathrm{n}:$ nombre de tranches & 10 & \\
\hline $\begin{array}{l}D_{23}: \text { diamètre intérieur du } \\
\text { tube }\end{array}$ & $0,015 \mathrm{~m}$ & \\
\hline $\begin{array}{l}D_{\text {ext }}: \text { diamètre extérieur du } \\
\text { tube }\end{array}$ & $0,017 \mathrm{~m}$ & \\
\hline $\boldsymbol{L}_{t u}:$ longueur du tube & $1,8 \mathrm{~m}$ & \\
\hline$S_{2}$ : surface de l'absorbeur & $2,07 \mathrm{~m}^{2}$ & \\
\hline$e_{1}:$ épaisseur de la vitre & $0,004 \mathrm{~m}$ & \\
\hline $\begin{array}{l}e_{2}: \text { épaisseur de } \\
\text { l'absorbeur }\end{array}$ & $0,005 \mathrm{~m}$ & \\
\hline $\begin{array}{l}e_{i s h}: \text { épaisseur de l'isolant } \\
\text { arrière }\end{array}$ & $0,05 \mathrm{~m}$ & \\
\hline $\begin{array}{l}e_{l a}: \text { épaisseur de l'isolant } \\
\text { latéral }\end{array}$ & $0,02 \mathrm{~m}$ & \\
\hline $\begin{array}{l}\tau_{1}: \text { coefficient de } \\
\text { transmission de la vitre }\end{array}$ & 0,83 & \\
\hline$\varepsilon_{1}:$ émissivité du vitrage & 0,85 & \multirow{13}{*}{$\begin{array}{l}\text { (Souad., } \\
2010)\end{array}$} \\
\hline $\begin{array}{l}\varepsilon_{2}: \text { émissivité de } \\
\text { l'absorbeur }\end{array}$ & 0,09 & \\
\hline$\varepsilon_{i s l}$ : émissivité de l'isolant & 0,05 & \\
\hline $\begin{array}{l}\alpha_{1}: \text { coefficient } \\
\text { d'absorption de la vitre }\end{array}$ & 0,06 & \\
\hline $\begin{array}{l}\alpha_{2}: \text { coefficient } \\
\text { d'absorption de l'absorbeur }\end{array}$ & 0,63 & \\
\hline $\begin{array}{l}\rho_{c}: \text { masse volumique de } \\
\text { la vitre }\end{array}$ & $2700 \mathrm{Kg} / \mathrm{m}^{3}$ & \\
\hline $\begin{array}{l}\rho_{a b}: \text { masse volumique de } \\
\text { l'absorbeur }\end{array}$ & $2700 \mathrm{Kg} / \mathrm{m}^{3}$ & \\
\hline $\begin{array}{l}\rho_{i s l}: \text { masse volumique de } \\
\text { l'isolant }\end{array}$ & $40 \mathrm{Kg} / \mathrm{m}^{3}$ & \\
\hline $\begin{array}{l}c_{p c}: \text { chaleur massique de } \\
\text { verre }\end{array}$ & $840 \mathrm{~J} / \mathrm{Kg} . \mathrm{K}$ & \\
\hline $\begin{array}{l}c_{p a b}: \text { chaleur massique de } \\
\text { l'absorbeur }\end{array}$ & $879 \mathrm{~J} / \mathrm{Kg} . \mathrm{K}$ & \\
\hline $\begin{array}{l}c_{p i s l}: \text { chaleur massique de } \\
\text { l'isolant }\end{array}$ & $840 \mathrm{~J} / \mathrm{Kg} . \mathrm{K}$ & \\
\hline $\begin{array}{l}\lambda_{c}: \text { conductivité } \\
\text { thermique de verre }\end{array}$ & $\begin{array}{lll}0,93 & \mathrm{~W} \quad \mathrm{~m}^{-1} \\
\mathrm{~K}^{-1} & & \\
\end{array}$ & \\
\hline $\begin{array}{l}\lambda_{a b}: \text { conductivité } \\
\text { thermique de l'absorbeur }\end{array}$ & $\begin{array}{lll}204 & W & \mathrm{~m}^{-1} \\
\mathrm{~K}^{-1} & & \\
\end{array}$ & \\
\hline $\begin{array}{l}\lambda_{i s l}: \text { conductivité } \\
\text { thermique de l'isolant }\end{array}$ & $\begin{array}{l}0,041 \mathrm{~W} \mathrm{~m}^{-1} \\
\mathrm{~K}^{-1}\end{array}$ & \\
\hline $\begin{array}{l}\sigma: \text { constante de Stéphane } \\
\text { Boltzman }\end{array}$ & $\begin{array}{l}5,67 \times 10^{-8} \\
\mathrm{~W} / \mathrm{m}^{2} \mathrm{~K}^{4}\end{array}$ & \\
\hline
\end{tabular}

\subsection{Variation temporelle du rayonnement solaire}

Sur la figure 4, est représentée l'intensité du rayonnement solaire en fonction du temps enregistré le 01/01/2020 par la station radiométrique située sur le site de la faculté des sciences et techniques. On constate que l'intensité croit de $7 \mathrm{~h}$ à $11 \mathrm{~h}$, atteint son maximum entre $11 \mathrm{~h}$ et $14 \mathrm{~h}$, puis décroit.

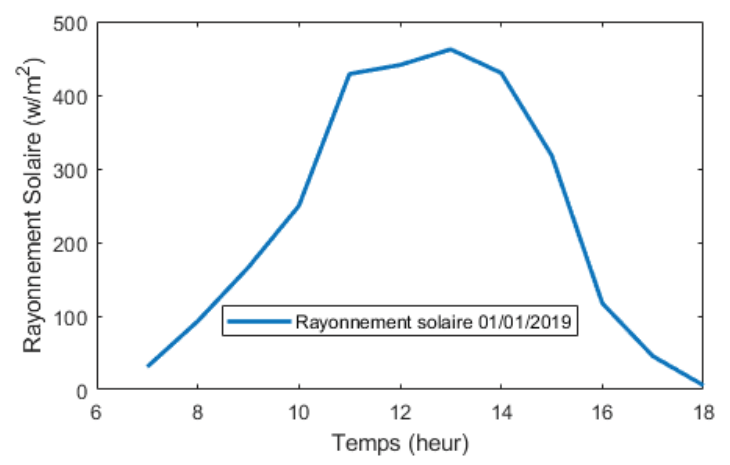

Fig.4. Variation de l'intensité solaire en fonction du temps

\subsection{Effet de la vitesse du vent sur la température} du fluide caloporteur et le rendement du capteur

La température du fluide caloporteur diminue légèrement avec l'accroissement de la vitesse du vent comme le montre la figure 5 . Le capteur se refroidi à cause de l'augmentation de l'échange par convection entre le capteur et l'ambiant.

L'augmentation de la vitesse du vent engendre aussi une baisse de la température des différents éléments du capteur. Ceci conduit à une élévation des pertes donc à un décroissement du rendement instantané du capteur (figure 6). Ainsi, lorsque la vitesse du vent varie de $1 \mathrm{~m} / \mathrm{s}$ à $4 \mathrm{~m} / \mathrm{s}$, le rendement instantané passe de $69 \%$ à $65 \%$ pour un rayonnement solaire de $460 \mathrm{~W} / \mathrm{m}^{2}$. Les vitesses de vent faible donnent un meilleur rendement (Zhang et al., 2015) et une température plus élevée du fluide à la sortie.

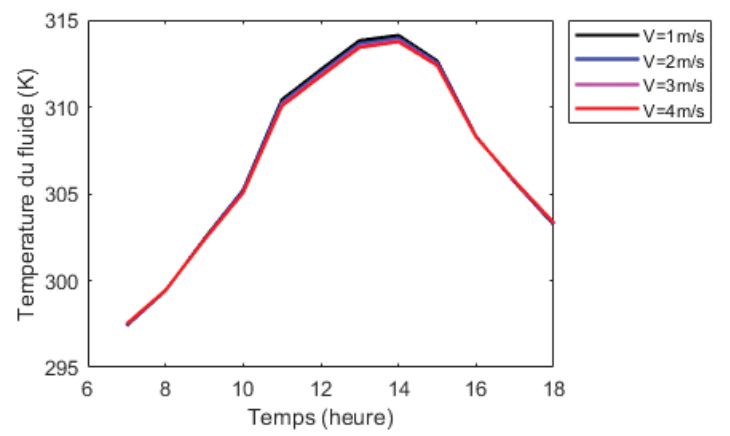

Fig.5. Evolution de la température du fluide caloporteur au cours de la journée.

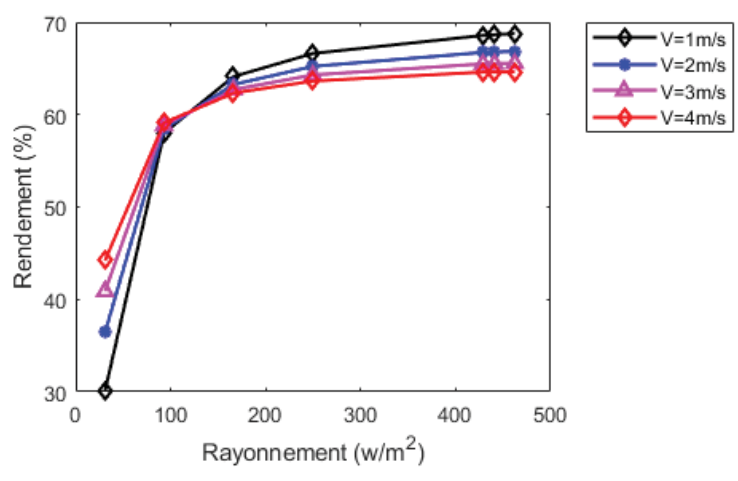

Fig.6. Evolution du rendement en fonction du rayonnement pour différentes valeurs de la vitesse du vent. 


\subsection{Effet de l'épaisseur de l'isolant sur la température du fluide caloporteur et le rendement du capteur}

Il apparait, sur la figure 7 que la température du fluide augmente légèrement en fonction de l'accroissement de l'épaisseur de l'isolant dû principalement à la diminution des pertes par conduction.

L'influence de l'épaisseur de l'isolant sur le rendement est illustrée par la figure 8. Une augmentation de l'épaisseur de l'isolant entraine de faibles pertes par conduction à l'arrière du capteur conduisant à un rendement légèrement élevé en accord avec les résultats de Zhang et al., 2015. Le rendement passe de $43 \%$ à 60 $\%$ lorsque le rayonnement solaire varie de 30 à $100 \mathrm{~W} / \mathrm{m}^{2}$. A partir de $200 \mathrm{~W} / \mathrm{m}^{2}$, la différence de rendement correspondant aux épaisseurs extrêmes de l'isolant $(5 \mathrm{~cm}$ $14 \mathrm{~cm}$ ) ne dépasse pas 2,5\%. Les valeurs maximum et minimum du rendement ( $66 \%$ et de $63,5 \%$ ) pour une intensité solaire de $460 \mathrm{~W} / \mathrm{m}^{2}$ correspondent respectivement aux épaisseurs $14 \mathrm{~cm}$ et $5 \mathrm{~cm}$.

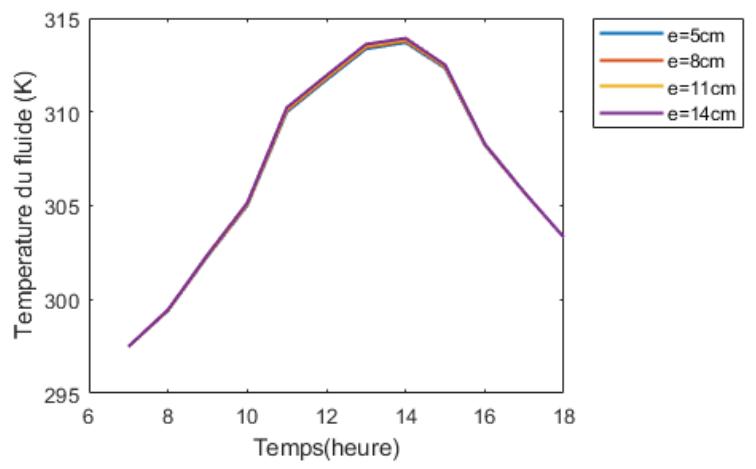

Fig. 7. Evolution de la température du fluide caloporteur au cours de la journée.

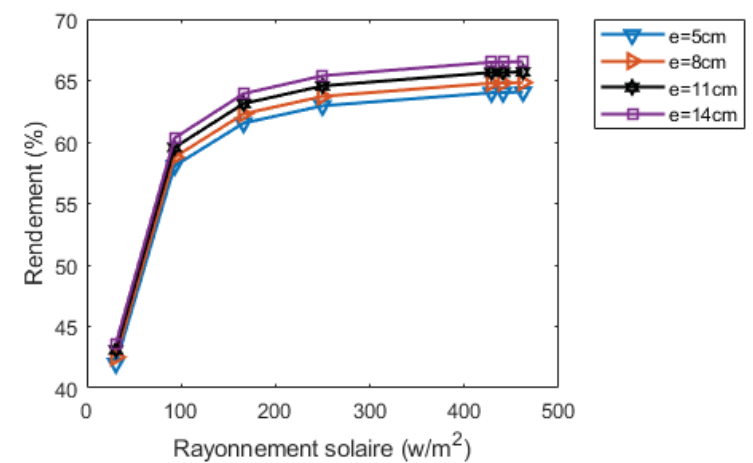

Fig.8. Evolution du rendement en fonction du rayonnement pour différentes valeurs de l'épaisseur.

\subsection{Influence des dimensions des tubes sur le rendement et la température du capteur}

L'augmentation des diamètres extérieur et intérieur des tubes de l'absorbeur conduit à l'accroissement du rendement comme l'indique la figure 9 et les résultats de la littérature (Souad., 2010; Zhang et al., 2015). La variation de la taille des tubes implique un nombre de Reynolds plus élevé, ce qui favorise le transfert convectif entre l'absorbeur et le fluide d'où une élévation de rendement. Une augmentation de $1 \mathrm{~mm}$ des diamètres entraine une augmentation du rendement d'environ $4 \%$.
Quant à la température, les diamètres des tubes utilisés ont très peu d'influence sur elle (figure 10).

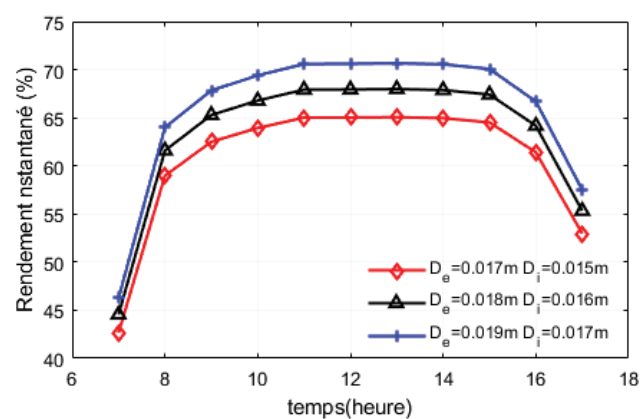

Fig.9. Evolution journalière du rendement instantané pour différentes valeurs des dimensions des tubes.

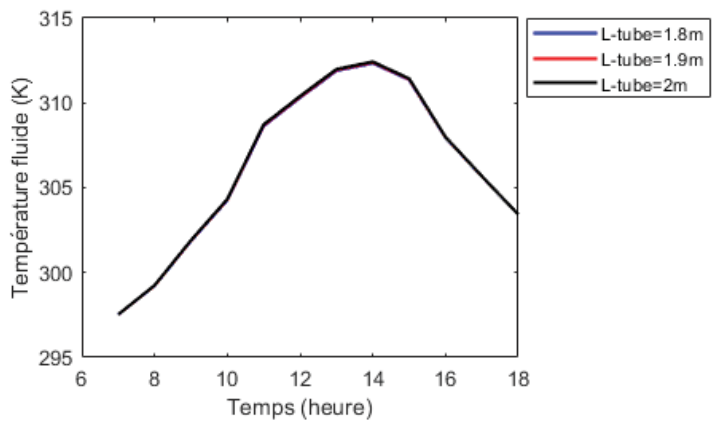

Fig.10. Evolution journalière de la température correspondant à différentes valeurs des dimensions des tubes.

\subsection{Influence de la nature du matériau de l'absorbeur sur la température du fluide caloporteur et le rendement.}

Comme nous pouvons le constater sur la figure 12 , le rendement varie substantiellement (49\%, $59 \%$ et $71 \%)$ en fonction de la nature du matériau (aluminium, cuivre et acier chrome-noir). Il est quasi constant pour un rayonnement solaire d'une intensité supérieure à 100 $\mathrm{W} / \mathrm{m}^{2}$. Cela est dû à la faible émissivité de l'acier chrome-noir qui réduit les pertes radiatives et à sa forte absorptivité dû à sa couche sélective. L'impact du matériau sur la température du fluide caloporteur est de 1 $\mathrm{K}$ entre une plaque absorbante en acier chrome-noir et le cuivre, de même qu'entre le cuivre et l'aluminium (figure 12).

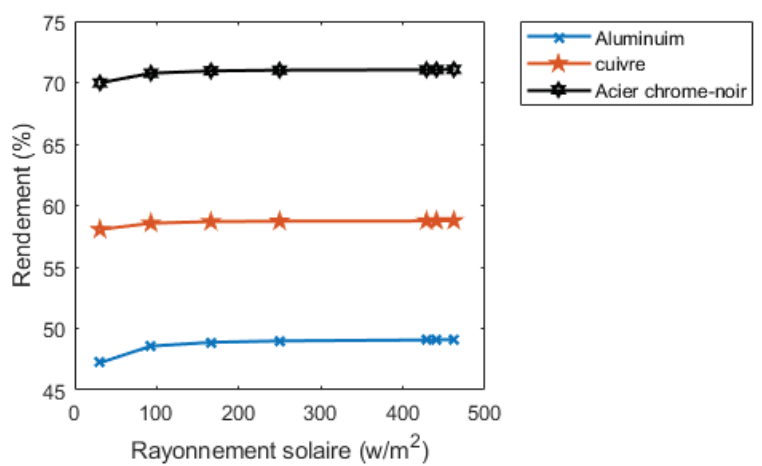

Fig.11. Évolution du rendement en fonction du rayonnement pour différents types de matériau. 


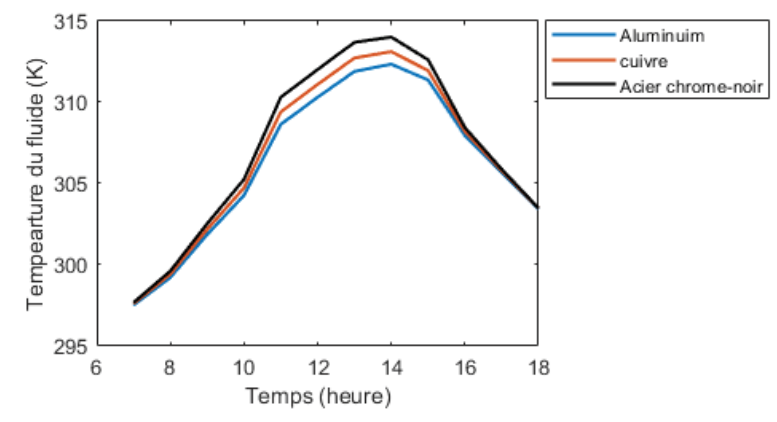

Fig.12. Evolution de la température du fluide caloporteur au cours de la journée en fonction du rayonnement pour différents types de matériau.

\subsection{Influence de la nature du matériau de la vitre sur la température du fluide caloporteur et le rendement instantané}

L'évolution du rendement et de la température du fluide caloporteur du capteur en fonction des propriétés optiques de la vitre (couverture transparente) est présentée par les figures 13 et 14. On n'observe quasiment pas de différence de température du fluide caloporteur avec la nature des différentes couvertures transparentes utilisées dans cette étude (figure13).

Pour une intensité solaire variant de $30 \mathrm{~W} / \mathrm{m}^{2}$ à $200 \mathrm{~W} / \mathrm{m}^{2}$, le rendement instantané du capteur passe respectivement de $57,2 \%, 58 \%, 58,8 \%$ et $59,5 \%$ à $59 \%, 59,8 \%, 60,5$ $\%$ et $62 \%$ pour le verre clair, le plastique plexiglas, le verre primé et le poly-méthacrylate. Le rendement est quasi constant au-delà de $200 \mathrm{~W} / \mathrm{m}^{2}$.

La température et le rendement de la couverture transparente en poly-méthacrylate sont légèrement plus élevés que ceux en verre primé, en plexiglas ou en verre claire

Ce résultat s'explique par la forte transmissivité de cette couverture face aux rayons solaires de petite longueur d'onde et sa faible émissivité qui réduit les pertes radiatives.

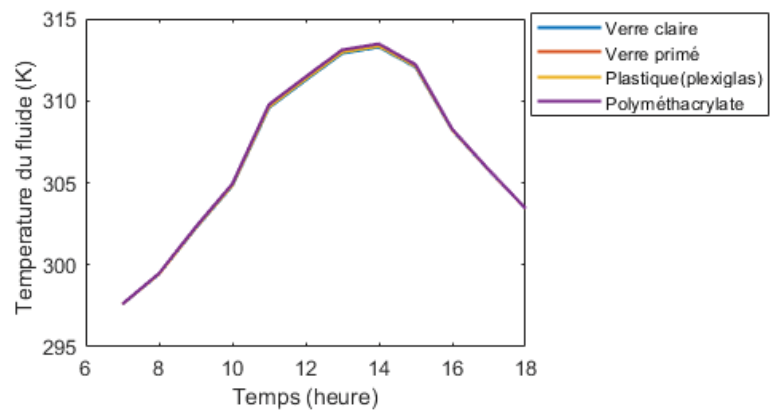

Fig.13. Evolution de la température du fluide caloporteur au cours de la journée.

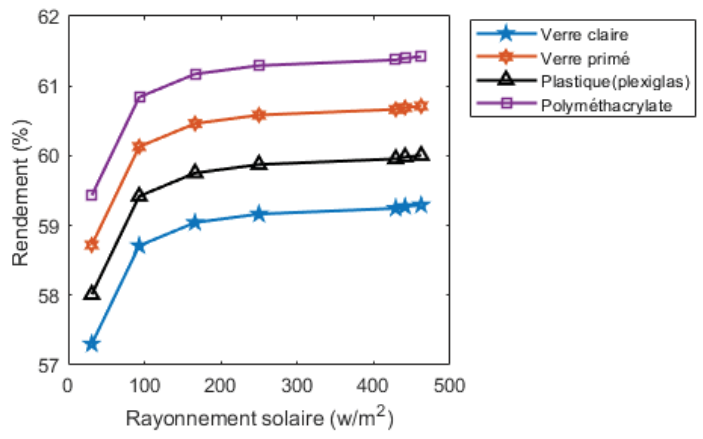

Fig.14. Evolution du rendement en fonction du rayonnement pour différents types de vitrage.

\subsection{Influence de la nature du matériau de l'isolant sur la température du fluide caloporteur et le rendement instantané.}

Les figures 15-16 montrent les effets des différents matériaux qui peuvent être utilisés comme isolant sur le rendement instantané et la température du fluide caloporteur. Comme nous pouvons l'observer sur la figure 15, la nature du matériau utilisé n'a pratiquement aucun impact sur la variation de la température. La mousse de polyuréthane comme isolant (figure 16), présente le meilleur rendement ( 52\%) par rapport aux autres types d'isolant $(\sim 49,5 \%, \sim 50,4 \%)$ due à ses propriétés thermo-physiques telles que la faible conductivité thermique et une importante chaleur spécifique.

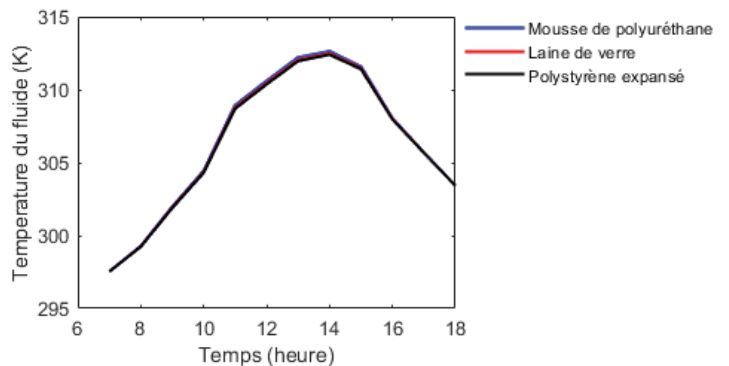

Fig.15. Evolution de la température du fluide caloporteur au cours de la journée

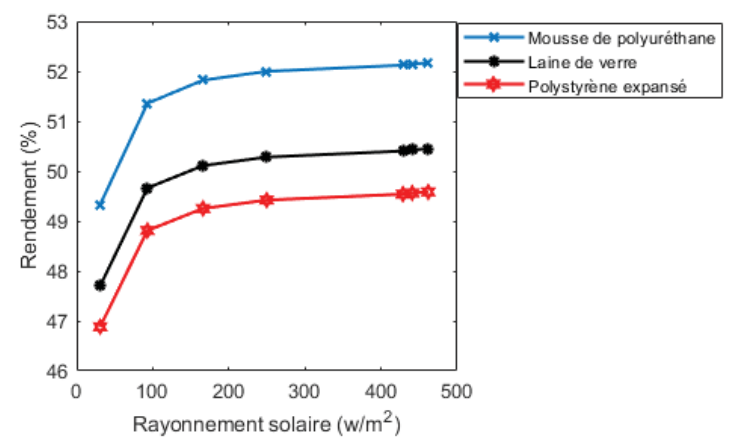

Fig.16. Évolution du rendement en fonction du rayonnement pour différents types d'isolant.

\subsection{Influence de la longueur du tube sur le rendement instantané et la température de sortie}

Lorsque la longueur du tube passe de 1,8 m à $2 \mathrm{~m}$, le rendement instantané augmente d'un peu plus de $1 \%$ (figure 17) pour une intensité variant de $30 \mathrm{~W} / \mathrm{m}^{2}$ à 200 $\mathrm{W} / \mathrm{m}^{2}$. À partir de $200 \mathrm{~W} / \mathrm{m}^{2}$, pour les longueurs simulées le rendement est constant et augmente de $0,5 \%$. En effet, 
plus le tube est long, plus le temps du trajet du fluide augmente. De ce fait, l'écart entre la température du fluide à l'entrée et la température à la sortie augmente, ce qui implique une légère augmentation du rendement. Aussi, l'augmentation de la longueur du tube passant de $1,8 \mathrm{~m}$ à $2 \mathrm{~m}$ influence très peu la variation de la température comme nous pouvons l'observer sur la figure 18.

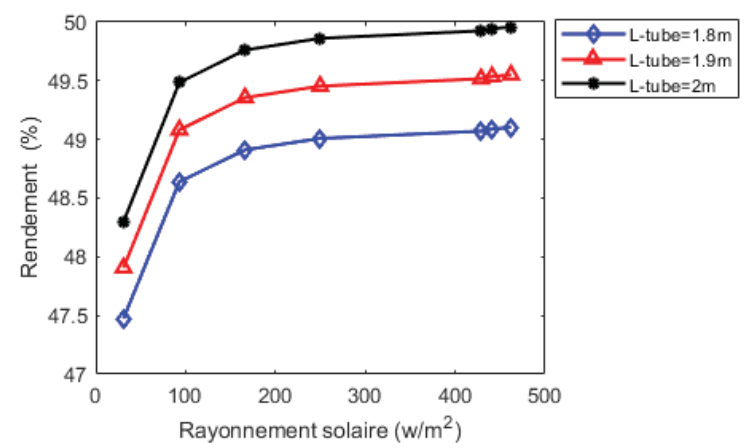

Fig.17. Effet de la longueur du tube sur le rendement instantané

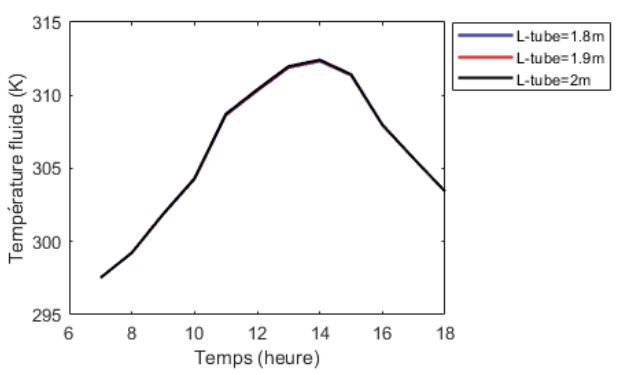

Fig.18. Effet de la longueur du tube sur la température

\section{CONCLUSION}

Cette étude a permis d'élaborer un modèle numérique, de simuler quelques paramètres externes (rayonnement solaire, la vitesse du vent) et internes (propriétés optiques et thermo-physiques des différents composants du capteur) influant sur le rendement et la température du capteur solaire plan au mois de Janvier sous les conditions météorologiques du Mali. Les résultats ont montré que pour un rayonnement solaire maximum de $460 \mathrm{~W} / \mathrm{m}^{2}$ :

- le rendement diminue de $69 \%$ à $65 \%$, lorsque la vitesse du vent croit de $1 \mathrm{~m} / \mathrm{s}$ à $4 \mathrm{~m} / \mathrm{s}$;

- $\quad$ lorsque l'épaisseur de l'isolant passe de $5 \mathrm{~cm}$ à 14 $\mathrm{cm}$, le rendement augmente de $64 \%$ à $67 \%$ respectivement ;

- l'augmentation des diamètres intérieur et extérieur des tubes, entraine celle du rendement dont la valeur maximale atteinte est de $70 \%$;

- pour un absorbeur en acier chrome, le rendement obtenu est maximale (71\%).

- Le rendement le plus élevé est de $61,5 \%$ pour une vitre en poly-méthacrylate.

- L'isolant dont le matériau est constitué de mousse de polyuréthane fourni le rendement le plus élevé soit $52,5 \%$.
- Plus le tube dans lequel, le fluide caloporteur circule est long, plus le rendement est élevé, il atteint 50\% dans le cas de notre étude.

Enfin la température du fluide caloporteur à la sortie varie peu en fonction des paramètres internes et externes étudiés, sa valeur est comprise entre $312,5 \mathrm{~K}$ et $314 \mathrm{~K}$.

\section{REMERCIEMENTS}

Nous remercions l'ISP (International Science Programme) pour son soutien.

\section{REFERENCES}

Benkhelifa, A., 2098. Optimisation d'un capteur solaire plan. Rev.Energ.Ren: Physique Energétique, pp.13-18.

Brahimi, A., 2016. Etude de la performance d'un capteur solaire plan à eau. Génie des procédés. Hal_01825548 .

Duffie, John. A et William, A Beckman., 2013. Solar Engineering of Thermal processes. John Wiley \& Sons, Interscience, New York, USA.

Hhedim, A.H., 2003. Energie solaire et son utilisation sous forme thermique et photovoltaïque. Centre de Publication Universitaire.

Hollands, K. G. T., et al., 2076. Free Convective Heat Transfer Across Inclined Air Layers. Journal of heat transfert", 98(2), pp.189-203.

Matlab., R2020a. Natick, Massachusetts: The MathWork

McADAMS, W. H., 2054. Heat Transmission. Sidney D. Kirkpatrick, Consulting Editor.

Ministère de l'énergie et de l'eau, Direction nationale de l'énergie., 2020. Prospectus d'investissement de l'énergie durable pour tous SEforALL du MALI. https://www.se4all-

africa.org/fileadmin/uploads/se4all/Documents/Countr y_AAs/PI_SEforALL_MALI.pdf

Nedjemeddine, N., 2018. Étude des performances d'un capteur solaire plan à conversion thermique. Mémoire en énergétique et environnement. Université Badji Mokhtar Annaba.

Sandali, M. et A. N Korti., 2016. Amélioration des performances thermiques $d u$ capteur solaire. $10^{\text {ème }}$ Journée de Mécanique de l'EMP (JM 10-EMP).

Selmi, M et al., 2008. Validation of CFD simulation for flat plate solar energy collector. Renew. Energy 33, pp.383-387.

Souad, S., 2010. Effet des parametres operationnels sur les performances d'un capteur solaire plan. Magistère en physique, Université Mentouri de Constantine.

Zhang Jiandong et al., 2015. Numerical simulation for structural parameters of flat-plate solar collector. Solar Energy 117, pp.202-202. 